\title{
Calculations of Energy Band Structure of GaAs, GaSb and GaP Crystals as a Function of Temperature Using the Semiempirical Tight Binding Method
}

\author{
Ismail Th. T. Yahya ${ }^{1 *}$, Mumtaz M. S. Hussien ${ }^{2}$ \\ $1^{*, 2}$ Department of Physics, College of Education for Pure Sciences, University of Mosul, Mosul, Iraq \\ E-mail: ${ }^{1 *}$ ismael.esp126@student.uomosul.edu.iq, ${ }^{2}$ momtaz_hussien@uomosul.edu.iq
}

(Received June 28, 2021; Accepted August 23, 2021; Available online September 01, 2021)

DOI: 10.33899/edusj.2021.130682.1170, (c) 2021, College of Education for Pure Science, University of Mosul.

This is an open access article under the CC BY 4.0 license (http://creativecommons.org/licenses/by/4.0/).

\begin{abstract}
In this paper, the band structure of gallium group of III-V semiconductor has been calculated with temperature, the semi-empirical tight binding method was used to calculate the band structure and the matrix elements were calculated for both models $\mathrm{sp}^{3}$ and $\mathrm{sp}^{3} \mathrm{~s}^{*}$. A computer program in MATLAB was designed to calculate the energy eigenvalues for the wave vector points in the first Brillouin zone between high symmetry points to form energy bands. The effect of temperature on the energy band of these group of crystals has been studied by changing the values of the lattice constant under the influence of temperature according to the Pierron relation and thus calculating the change in the length of the bond with temperature, which in turn affects the change in the values of the elements of the Hamiltonian matrix. The energy gap and refractive index were calculated at points of high symmetry as a function of temperature. The results showed a decrease in the energy gap values of $\mathrm{GaAs}, \mathrm{GaSb}$ and GaP crystals with increasing temperature. Then the experimental Varshni relation was used to calculate the change in the energy gap values of these crystals and the results obtained from current study were compared with the results of Panish and Bellani, where the results showed that a model $\mathrm{sp}^{3} \mathrm{~s}^{*}$ gives better results than the model $\mathrm{sp}^{3}$.As well as the calculations of the refractive index of these crystals using the Moss formula showed that the refractive index will gradually increase with increasing temperatures.
\end{abstract}

Keyword: energy bands, semiconductor bands. energy gap with temperature, refractive index.

$$
\begin{aligned}
& \text { حسابات تركيب حزم الطاقة للبلورات GaAs، GaSb وGaP كدالة لارجة الحرارة بأستخدام طريقة الربط } \\
& \text { المحكم شبه التجريبية } \\
& \text { اسماعيل ثمين طليع يحيى 1*، ممتاز محمد صالح حسين } 2 \\
& \text { 1*2،قسم الفيزياء، كلية التربية للعلوم الصرفة، جامعة الموصل، الموصل، العر اق }
\end{aligned}
$$

\footnotetext{
الملخص:

لقد أحتسب في هذا البحث تركيب حزم الطاقة لمجمو عة الكاليوم في بلور ات شبه الموصل من نوع III-V مع درجة الحر ارةة،

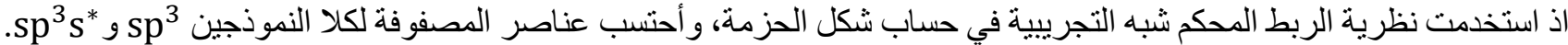

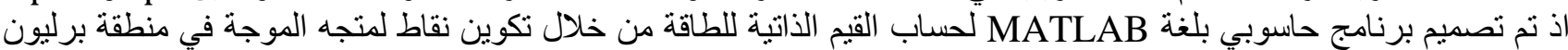

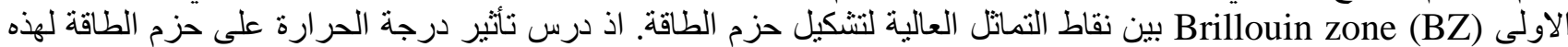

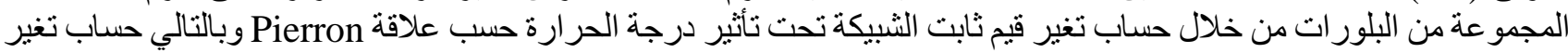
طول الآصرة مع درجة الحر ارة و التي تؤثر بدور ها في تغير قيم معاملات عناصر مصفوفة الهاملتوني. تم حساب فجوة الطاقة ومعامل
} 


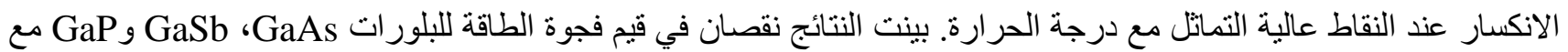

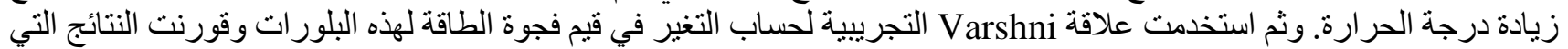

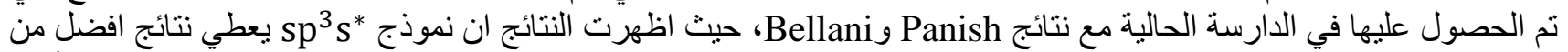

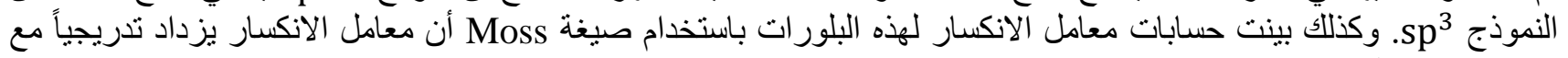
زيادة درجات الحرارة.

الكلمات المفتاحية: حزم الطاقة، حزم اشباه الموصلات، فجوة الطاقة مع درجة الحر ارة، معامل الانكسار.

Introduction 1 المقدمة

تعد درجة الحرارة والضغط من أهم المتغيرات الثرموداينميكية عند دراسة تراكيب حزم الطاقة لأشباه الموصلات المختلفة. اذ بذل الكثير من الباحثين جهوداً كبيرة لاستغلال الخصائص الحرارية لأشباه الموصلات تحت تأثير درجات الحرارة العالية لأنها ذات أهمية أساسية للتطبيقات التكنولوجية [1]. فعند زيادة درجة حرارة المواد شبه الموصلة تحدث تغيرات بالتركيب الالكتروني وبدراسة هذه التغيرات يمكن اجراء العديد من التعديلات في تركيب الحزمة، اذ يؤثر ارتفاع درجة الحرارة في فجوة الطاقة وبالتالي يحدث تغيير في الكتل الفعالة لكل من الالكترون والفجوة.لذلك فإن دراسة تغيرات فجوة الطاقة مع درجة الحرارة أمر ضروري لأشباه الموصلات. اذ يمكن من خلال دراسة تأثير درجات الحرارة العالية على تركيب حزم الطاقة لمواد شبه الموصلة من معرفة فجوة الطاقة التي تلعب دوراً مهماً للغاية في أشباه الموصلات لأنها ستؤثر بشكل كبير على الخصائص الكهربائية لمواد ونبائط أشباه الموصلات [2]. تعد

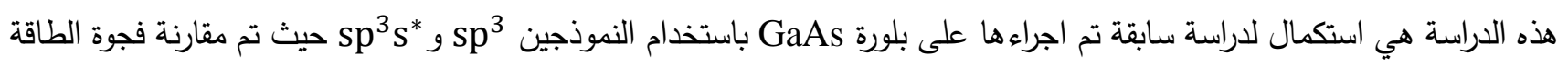
في كلا النموذجين اضافة الى حساب الكتلة الفعالة في الاتجاه [111] بشكل مباشر من حساب حزم الطاقة [3]. يهدف البحث الى تطبيق طريقة الربط المحكم للجيران الاقرب بأستخدام النموذجين sp³ s sp لحساب تركيب حزم الطاقة مع درجة الحرارة للبلورات GaP و GaSb GaAs وذلك من خلال اعداد برنامج حاسوبي وحساب تغير فجوة الطاقة مع درجات حرارة مختلفة عند نقاط التماثل العالية بدون استخدام معادلة Varshni وباستخدامها وحساب تغير معامل الانكسار بأستخدام صيغة Moss عند درجات حرارة

مختلفة.

\section{2}

يقلل التتاظر في المواد البلورية العديد من المشاكل التي تتعرض لها وحدة الخلية الابتدائية، ويمكن كتابة دوال

الموجة للإلكترون المنفرد $|n \vec{k}|$ على شكل موجات بلوخ [4]:

$$
|n \vec{k}\rangle=\sum_{\alpha \beta} C_{\alpha, \beta}(n, \vec{k})\left\{\sum_{R} e^{\mathrm{i} \vec{k} . \vec{R}_{\beta}} \mid \alpha, \vec{R}_{\beta}\right\}
$$

اذ ان التوالي. في حين تمثل المعاملات لأثباه الموصلات ذات تركيب المشبك الخارصيني (ZB) فأن نموذج الربط المحكم sp الذي قدمه Chadi بدون تأثير تفاعل البرم 
- المدار يستخدم حالة واحدة من المدار s وثلاث حالات من المدار p للذرة الاساس بالتالي تتتج 8 مدارات لخلية بدائية تحتوي على

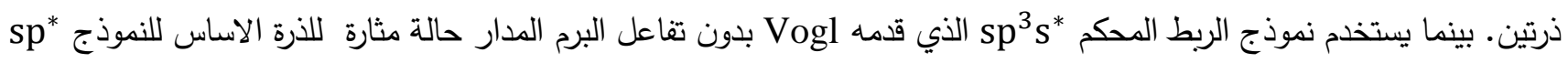

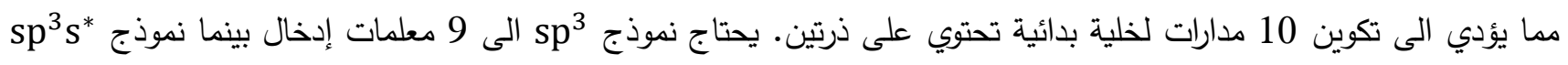
يحتاج الى 13 من معلمات الادخال والتي يتم تحديدها بالمقارنة مع نتائج تجريبية [5, 6]. معلمات التفاعل هذه عبارة عن عناصر مصفوفة مستقلة بشكل أساسي، تتكون من اربع طاقات هي

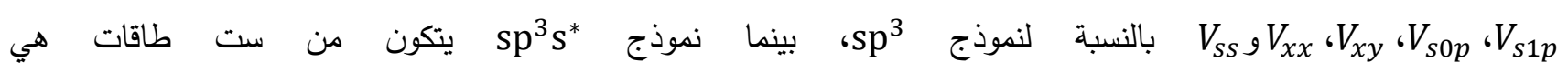

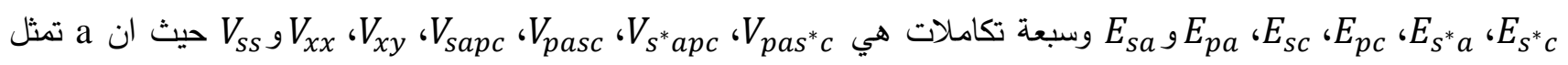
الأنيون وc تمثل الكاتيون [7].

\section{Results and calculations 3.}

يكون تأثير الحرارة على مواد اشباه الموصلات من خلال التعديل في الترتيب الذري، والتي تشمل التغير في أطوال الاواصر الذرية d وكذلك الزاويا للتراكيب المكعبة مثل تركيب ZB [8]. اذ تم أدخال تأثير درجة الحرارة في حسابات عناصر المصفوفة من خلال تغير ثابت الثبيكة مع درجة الحرارة حسب علاقة Pierron والتي تعطى بالصيغة الاتية [9]:

$$
\alpha_{t h}=\frac{1}{a} \frac{\partial a}{\partial T}
$$

ومن خلال هذه العلاقة وبعد معرفة قيم معامل التمدد الحراري مh للبلورات GaP و GaSb GaAs المبينة في الجدول (1) نستتتج قيمة ثابت الثبيكة عند درجة الحرارة معينة $a_{T}$ اذ يعطى بالعلاقة الاتية:

$$
a_{T}=a_{0} e^{\alpha_{t h} T}
$$

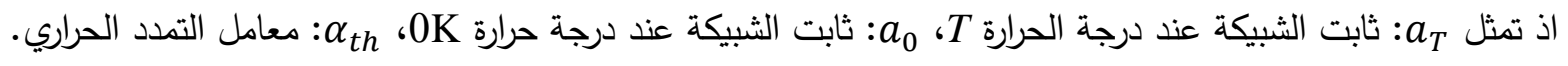

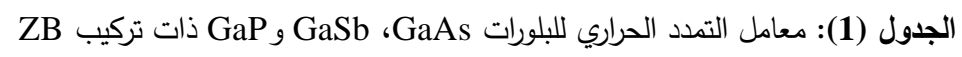

\begin{tabular}{|c|c|c|}
\hline Crystal & $\alpha_{t h} \times 10^{-6}(1 / \mathrm{K})$ & Ref. \\
\hline $\mathrm{GaAs}$ & 5.73 & {$[10]$} \\
\hline $\mathrm{GaSb}$ & 6.1 & {$[10]$} \\
\hline $\mathrm{GaP}$ & 6.1 & {$[11]$} \\
\hline
\end{tabular}

ومن تغير ثابت الشبيكة مع درجة الحرارة يتم حساب قيم طول الآصرة تحت تأثير درجة الحرارة باستخدام العلاقة التالية [12]:

$$
\begin{aligned}
& d=\frac{\sqrt{3}}{4} a \\
& d_{0}=\frac{\sqrt{3}}{4} a_{0} \\
& d_{T}=\frac{\sqrt{3}}{4} a_{T}
\end{aligned}
$$

اذ يمثل d.

اذ يمثل Td: طول الآصرة بين الذرات عند درجة الحرارة T. ومن خلال المعادلتين (5) و (6) يمكن استتتاج العلاقة الاتية لحساب قيم طول الآصرة عند درجة حرارة معينة : 


$$
d_{T}=d_{0}\left(\frac{a_{T}}{a_{0}}\right)
$$

يبين الجدول (2) قيم طول الآصرة وثابت الثبيكة تحت تأثير درجة الحرارة بوحدات (K) للبلورات GaAs، GaSb و GaP.

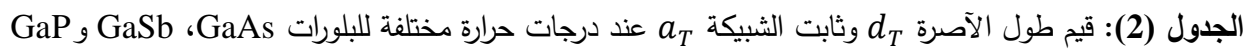

\begin{tabular}{|c|c|c|c|c|c|c|c|}
\hline \multirow{2}{*}{ Crystal } & \multirow{2}{*}{ Parameter } & \multicolumn{6}{|c|}{ Temperature (K) } \\
\hline & & 0 & 90 & 185 & 294 & 673 & 973 \\
\hline \multirow{2}{*}{ GaAs } & $a_{T}(\AA)$ & $5.6532[13]$ & 5.6562 & 5.6592 & 5.6628 & 5.6751 & 5.6849 \\
\hline & $d_{T}(\AA)$ & 2.4479 & 2.4492 & 2.4505 & 2.4520 & 2.4574 & 2.4616 \\
\hline \multirow{2}{*}{ Crystal } & \multirow{2}{*}{ Parameter } & \multicolumn{6}{|c|}{ Temperature $(\mathrm{K})$} \\
\hline & & 0 & 60 & 120 & 183 & 240 & 300 \\
\hline \multirow{2}{*}{$\mathrm{GaSb}$} & $a_{T}(\AA)$ & 6.0959 [13] & 6.0982 & 6.1004 & 6.1027 & 6.1049 & 6.1071 \\
\hline & $d_{T}(\AA)$ & 2.6396 & 2.6406 & 2.6415 & 2.6525 & 2.6435 & 2.6444 \\
\hline \multirow{2}{*}{ Crystal } & \multirow{2}{*}{ Parameter } & \multicolumn{6}{|c|}{ Temperature (K) } \\
\hline & & 0 & 150 & 417 & 620 & 868 & 1273 \\
\hline \multirow{2}{*}{$\mathrm{GaP}$} & $a_{T}(\AA)$ & $5.4505[13]$ & 5.4555 & 5.4644 & 5.4712 & 5.4794 & 5.4930 \\
\hline & $d_{T}(\AA)$ & 2.3601 & 2.3623 & 2.3661 & 2.3690 & 2.3726 & 2.3785 \\
\hline
\end{tabular}

بعد حساب التغيرات في طول الآصرة وثابت الثبيكة عند درجات حرارة مختلفة، تم حساب تغير تركيب حزم الطاقة عند درجة حرارة معينة من خلال تغير معاملات عناصر المصفوفة بواسطة قاعدة القياس التجريبية [14]:

$$
\begin{aligned}
V_{l l^{\prime} m} & =\eta_{l l^{\prime} m} \frac{\hbar^{2}}{2 m_{e} d^{2}} \\
V(0) & =\eta_{l l^{\prime} m}\left(\frac{\hbar^{2}}{2 m_{e} d_{0}^{2}}\right)
\end{aligned}
$$

اذ ان (0) V: تمثل معاملات الربط المحكم عند درجة حرارة 0K، و $V(T)=\eta_{l l^{\prime} m}\left(\frac{\hbar^{2}}{2 m_{e} d_{T}^{2}}\right)$

اذ ان (T) V(T: تمثل معاملات الربط المحكم عند درجة الحرارة T. ومن خلال المعادلتين (9) و (10) يمكن استتتاج علاقة لحساب تغير معاملات عناصر المصفوفة:

$$
V(T)=V(0)\left(\frac{d_{0}}{d_{T}}\right)^{2}
$$

وبعد ادخال درجة الحرارة في تغير قيم ثابت الشبيكة وطول الآصرة مع درجة الحرارة والتي بدورها تؤثر في معاملات عناصر المصفوفة التي تستخدم في حساب شكل حزم الطاقة، تم الحصول على نتائج حسابات عناصر المصفوفة عند درجات حرارة مختلفة للبلورات GaAs، GaSb و GaP وكما موضحة في الجداول (3)، (4) و (5) على التوالي. 
Journal of Education and Science (ISSN 1812-125X), Vol: 30, No: 4, 2021 (201-219)

الجدول (3): قيم عناصر المصفوفة عند درجات حرارة مختلفة لبلورة GaAs

\begin{tabular}{|c|c|c|c|c|c|c|c|}
\hline \multirow{2}{*}{ Model } & \multirow{2}{*}{$\begin{array}{c}\text { Matrix } \\
\text { Elements }\end{array}$} & \multicolumn{6}{|c|}{ Temperature(K) } \\
\hline & & 0 & 90 & 185 & 294 & 673 & 973 \\
\hline \multirow{9}{*}{$\mathrm{sp}^{3}$} & $E_{p 0}$ & 1.16 & 1.1588 & 1.1575 & 1.1561 & 1.1511 & 1.1471 \\
\hline & $E_{p 1}$ & 3.35 & 3.3465 & 3.3429 & 3.3387 & 3.3243 & 3.3129 \\
\hline & $E_{s 0}$ & -8.21 & -8.2015 & -8.1926 & -8.1824 & -8.1469 & -8.1190 \\
\hline & $E_{s 1}$ & -3.19 & -3.1867 & -3.1832 & -3.1793 & -3.1655 & -3.1546 \\
\hline & $V_{S S}$ & -6.76 & -6.7530 & -6.7457 & -6.7373 & -6.7081 & -6.6850 \\
\hline & $V_{x x}$ & 2.11 & 2.1078 & 2.1055 & 2.1029 & 2.0938 & 2.0866 \\
\hline & $V_{x y}$ & 5.96 & 5.9539 & 5.9474 & 5.9400 & 5.9142 & 5.8939 \\
\hline & $V_{s 0 p}$ & 4.75 & 4.7451 & 4.7399 & 4.7340 & 4.7135 & 4.6973 \\
\hline & $V_{s 1 p}$ & 5.48 & 5.4744 & 5.4684 & 5.4616 & 5.4379 & 5.4192 \\
\hline \multirow{13}{*}{$\mathrm{sp}^{3} \mathrm{~s}^{*}$} & $E_{s a}$ & -8.3431 & -8.3345 & -8.3254 & -8.3150 & -8.2790 & -8.2506 \\
\hline & $E_{p a}$ & 1.0414 & 1.0403 & 1.0392 & 1.0379 & 1.0334 & 1.0299 \\
\hline & $E_{s c}$ & 2.6569 & -2.6542 & -2.6513 & -2.6480 & -2.6365 & -2.6274 \\
\hline & $E_{p c}$ & 3.6686 & 3.6648 & 3.6608 & 3.6563 & 3.6404 & 3.6279 \\
\hline & $E_{s^{*} a}$ & 8.5914 & 8.5825 & 8.5732 & 8.5625 & 8.5254 & 8.4961 \\
\hline & $E_{s^{*} c}$ & 6.7386 & 6.7317 & 6.7243 & 6.7159 & 6.6868 & 6.6639 \\
\hline & $V_{S S}$ & -6.4513 & -6.4446 & -6.4376 & -6.4296 & -6.4017 & -6.3798 \\
\hline & $V_{x x}$ & 1.9546 & 1.9526 & 1.9505 & 1.9480 & 1.9396 & 1.9329 \\
\hline & $V_{x y}$ & 5.0779 & 5.0727 & 5.0671 & 5.0608 & 5.0389 & 5.0216 \\
\hline & $V_{\text {sapc }}$ & 4.4800 & 4.4754 & 4.4705 & 4.4649 & 4.4456 & 4.4303 \\
\hline & $V_{\text {pasc }}$ & 5.7839 & 5.7779 & 5.7717 & 5.7644 & 5.7395 & 5.7198 \\
\hline & $V_{s^{*} a p c}$ & 4.8422 & 4.8372 & 4.8318 & 4.8259 & 4.8050 & 4.7885 \\
\hline & $V_{\text {pas }^{*} c}$ & 4.8077 & 4.8027 & 4.7975 & 4.7915 & 4.7708 & 4.7544 \\
\hline
\end{tabular}


Journal of Education and Science (ISSN 1812-125X), Vol: 30, No: 4, 2021 (201-219)

الجدول (4): قيم عناصر عند درجات حرارة مختلفة لبلورة GaSb

\begin{tabular}{|c|c|c|c|c|c|c|c|}
\hline \multirow{2}{*}{ Model } & \multirow{2}{*}{$\begin{array}{c}\text { Matrix } \\
\text { Elements }\end{array}$} & \multicolumn{6}{|c|}{ Temperature(K) } \\
\hline & & 0 & 60 & 120 & 183 & 240 & 300 \\
\hline \multirow{9}{*}{$\mathrm{sp}^{3}$} & $E_{p 0}$ & 0.68 & 0.6795 & 0.6790 & 0.6785 & 0.6780 & 0.6775 \\
\hline & $E_{p 1}$ & 2.63 & 2.6281 & 2.6262 & 2.6241 & 2.6223 & 2.6204 \\
\hline & $E_{s 0}$ & -6.84 & -6.8350 & -6.8300 & -6.8247 & -6.8200 & -6.8150 \\
\hline & $E_{s 1}$ & -3.91 & -3.9071 & -3.9043 & -3.9013 & -3.8986 & -3.8957 \\
\hline & $V_{S S}$ & -6.06 & -6.0556 & -6.0511 & -6.0465 & -6.0423 & -6.0379 \\
\hline & $V_{x x}$ & 1.64 & 1.6388 & 1.6376 & 1.6363 & 1.6352 & 1.6340 \\
\hline & $V_{x y}$ & 4.32 & 4.3168 & 4.3137 & 4.3104 & 4.3074 & 4.3042 \\
\hline & $V_{s 0 p}$ & 5.55 & 5.5459 & 5.5419 & 5.5376 & 5.5338 & 5.5297 \\
\hline & $V_{s 1 p}$ & 4.76 & 4.7565 & 4.7530 & 4.7494 & 4.7461 & 4.7426 \\
\hline \multirow{13}{*}{$\mathrm{sp}^{3} \mathrm{~s}^{*}$} & $E_{s a}$ & -7.3207 & -7.3153 & -7.3100 & -7.3044 & -7.2993 & -7.2940 \\
\hline & $E_{p a}$ & 0.8554 & 0.8548 & 0.8541 & 0.8535 & 0.8529 & 0.8523 \\
\hline & $E_{s c}$ & -3.8993 & -3.8964 & -3.8936 & -3.8906 & -3.8879 & -3.8851 \\
\hline & $E_{p c}$ & 2.9146 & 2.9125 & 2.9103 & 2.9081 & 2.9061 & 2.9040 \\
\hline & $E_{s^{*} a}$ & 6.6354 & 6.6305 & 6.6257 & 6.6206 & 6.6160 & 6.6112 \\
\hline & $E_{s^{*} c}$ & 5.9846 & 5.9802 & 5.9758 & 5.9713 & 5.9671 & 5.9627 \\
\hline & $V_{S S}$ & -6.1567 & -6.1522 & -6.1477 & -6.1430 & -6.1387 & -6.1342 \\
\hline & $V_{x x}$ & 1.5789 & 1.5777 & 1.5766 & 1.5754 & 1.5743 & 1.5731 \\
\hline & $V_{x y}$ & 4.1285 & 4.1255 & 4.1225 & 4.1193 & 4.1164 & 4.1134 \\
\hline & $V_{\text {sapc }}$ & 4.9601 & 4.9565 & 4.9528 & 4.9490 & 4.9456 & 4.9420 \\
\hline & $V_{\text {pasc }}$ & 4.6675 & 4.6641 & 4.6607 & 4.6571 & 4.6539 & 4.6504 \\
\hline & $V_{s^{*} a p c}$ & 4.9895 & 4.9858 & 4.9822 & 4.9784 & 4.9749 & 4.9713 \\
\hline & $V_{\text {pas }^{*} c}$ & 4.2180 & 4.2149 & 4.2118 & 4.2086 & 4.2057 & 4.2026 \\
\hline
\end{tabular}


Journal of Education and Science (ISSN 1812-125X), Vol: 30, No: 4, 2021 (201-219)

الجدول (5): قيم عناصر المصفوفة عند درجات حرارة مختلفة لبلورة GaP

\begin{tabular}{|c|c|c|c|c|c|c|c|}
\hline \multirow{2}{*}{ Model } & \multirow{2}{*}{$\begin{array}{l}\text { Matrix } \\
\text { elements }\end{array}$} & \multicolumn{6}{|c|}{ Temperature(K) } \\
\hline & & 0 & 150 & 417 & 620 & 868 & 1273 \\
\hline \multirow{9}{*}{$\mathrm{sp}^{3}$} & $E_{p 0}$ & 1.28 & 1.2777 & 1.2735 & 1.2704 & 1.2665 & 1.2603 \\
\hline & $E_{p 1}$ & 3.82 & 3.8130 & 3.8006 & 3.7912 & 3.7798 & 3.7611 \\
\hline & $E_{s 0}$ & -7.60 & -7.5861 & -7.5614 & -7.5427 & -7.5199 & -7.4829 \\
\hline & $E_{s 1}$ & -2.72 & -2.7150 & -2.7062 & -2.6995 & -2.6913 & -2.6781 \\
\hline & $V_{s S}$ & -7.66 & -7.6460 & -7.6211 & -7.6023 & -7.5793 & -7.5420 \\
\hline & $V_{x x}$ & 2.26 & 2.2559 & 2.2485 & 2.2430 & 2.2362 & 2.2252 \\
\hline & $V_{x y}$ & 6.20 & 6.1887 & 6.1685 & 6.1533 & 6.1347 & 6.1045 \\
\hline & $V_{s 0 p}$ & 5.33 & 5.3203 & 5.3030 & 5.2898 & 5.2739 & 5.2479 \\
\hline & $V_{s 1 p}$ & 5.84 & 5.8293 & 5.8104 & 5.7960 & 5.7785 & 5.7500 \\
\hline \multirow{13}{*}{$\mathrm{sp}^{3} \mathrm{~s}^{*}$} & $E_{s a}$ & -81124 & -8.0976 & -8.0712 & -8.0513 & -8.0269 & -7.9874 \\
\hline & $E_{p a}$ & 1.1250 & 1.1229 & 1.1193 & 1.1165 & 1.1131 & 1.1077 \\
\hline & $E_{s c}$ & -2.1976 & -2.1936 & -2.1864 & -2.1810 & -2.1745 & -2.1637 \\
\hline & $E_{p c}$ & 4.1150 & 4.1075 & 4.0941 & 4.0840 & 4.0717 & 4.0516 \\
\hline & $E_{s^{*} a}$ & 8.5150 & 8.4994 & 8.4718 & 8.4508 & 8.4253 & 8.3838 \\
\hline & $E_{s^{*} c}$ & 7.1850 & 7.1719 & 7.1485 & 7.1309 & 7.1093 & 7.0743 \\
\hline & $V_{S S}$ & -7.4709 & -7.4572 & -7.4330 & -7.4146 & -7.3922 & -7.3558 \\
\hline & $V_{x x}$ & 2.1516 & 2.1477 & 2.1407 & 2.1354 & 2.1289 & 2.1184 \\
\hline & $V_{x y}$ & 5.1369 & 5.1275 & 5.1108 & 5.0982 & 5.0828 & 5.0577 \\
\hline & $V_{\text {sapc }}$ & 4.2771 & 4.2693 & 4.2554 & 4.2449 & 4.2320 & 4.2112 \\
\hline & $V_{\text {pasc }}$ & 6.3190 & 6.3074 & 6.2869 & 6.2714 & 6.2524 & 6.2216 \\
\hline & $V_{s^{*} a p c}$ & 4.6541 & 4.6456 & 4.6305 & 4.6190 & 4.6051 & 4.5824 \\
\hline & $V_{\text {pas }}{ }^{*}$ & 5.0950 & 5.0857 & 5.0691 & 5.0566 & 5.0413 & 5.0165 \\
\hline
\end{tabular}

توضح الاشكال (1)، (2)، (3)، (4)، (5) و (6) تركيب حزم الطاقة للبلورات GaAs، GaP و GaSb عند درجة حرارة

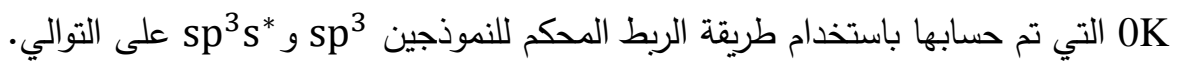




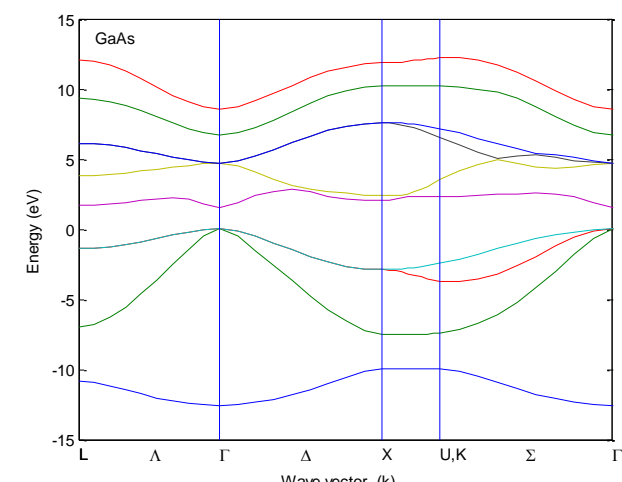

الثكل (4): تركيب حزمة الطاقة لبلورة GaAs

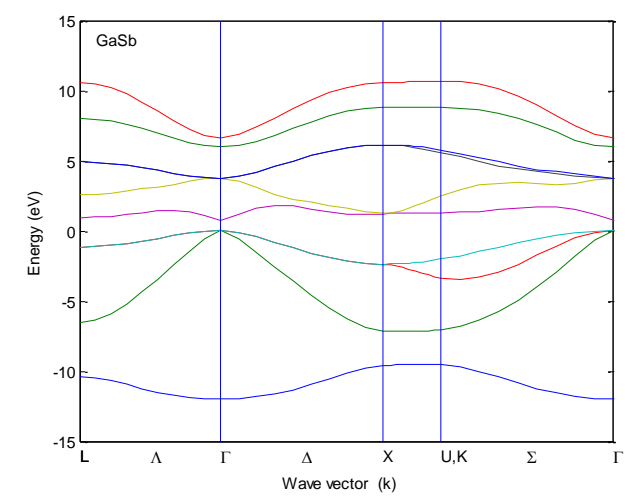

الثكل (5): تركيب حزمة الطاقة لبلورة GaSb

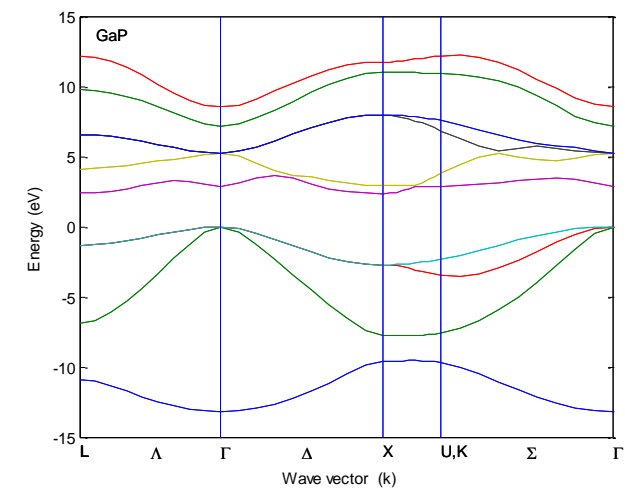

الثكل (6): تركيب حزمة الطاقة لبلورة GaP

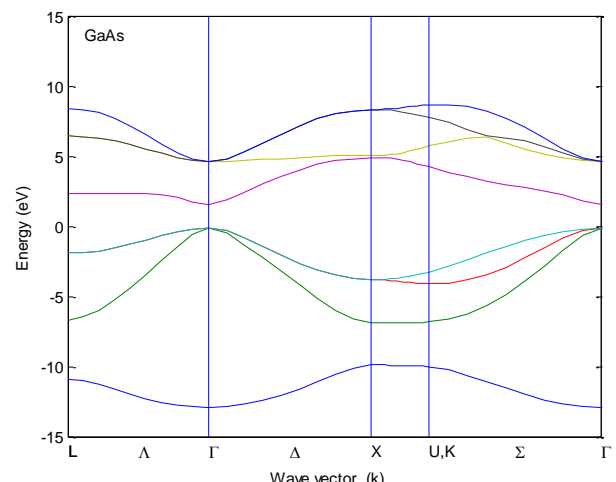

الثكل (1): تركيب حزمة الطاقة لبلورة GaAs لنموذج

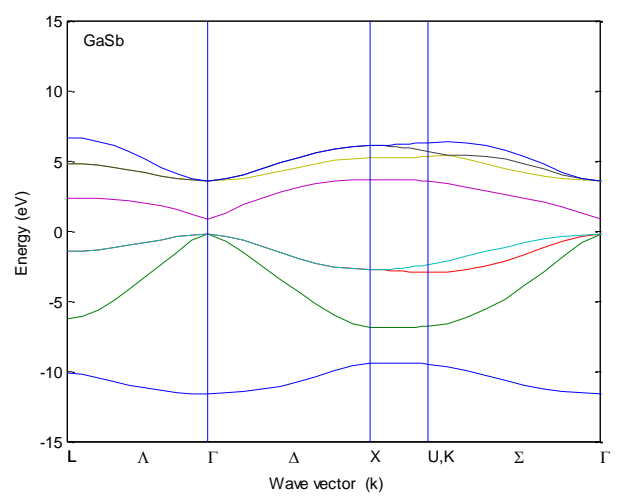

الثكل (2): تركيب حزمة الطاقة لبلورة GaSb

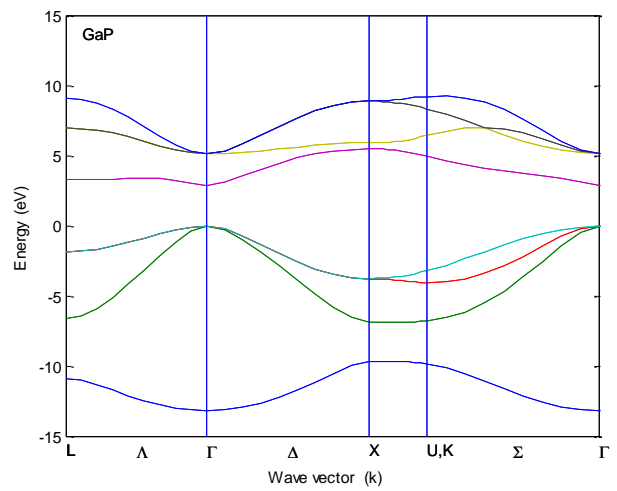

الثكل (3): تركيب حزمة الطاقة لبلورة GaP لنموذج

وعند استخدام معاملات عناصر المصفوفة عند درجات الحرارة العالية المختلفة لبلورات GaAs، GaSb و GaP المبينة في

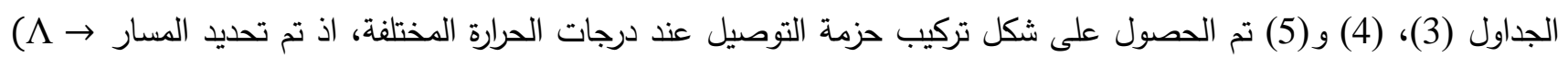

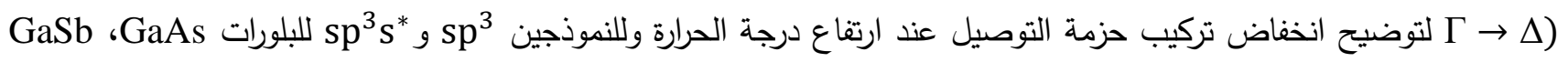
و GaP وكما موضحة في الاشكال (7)، (8)، (9)، (10)، (11) و (12) على التوالي. 

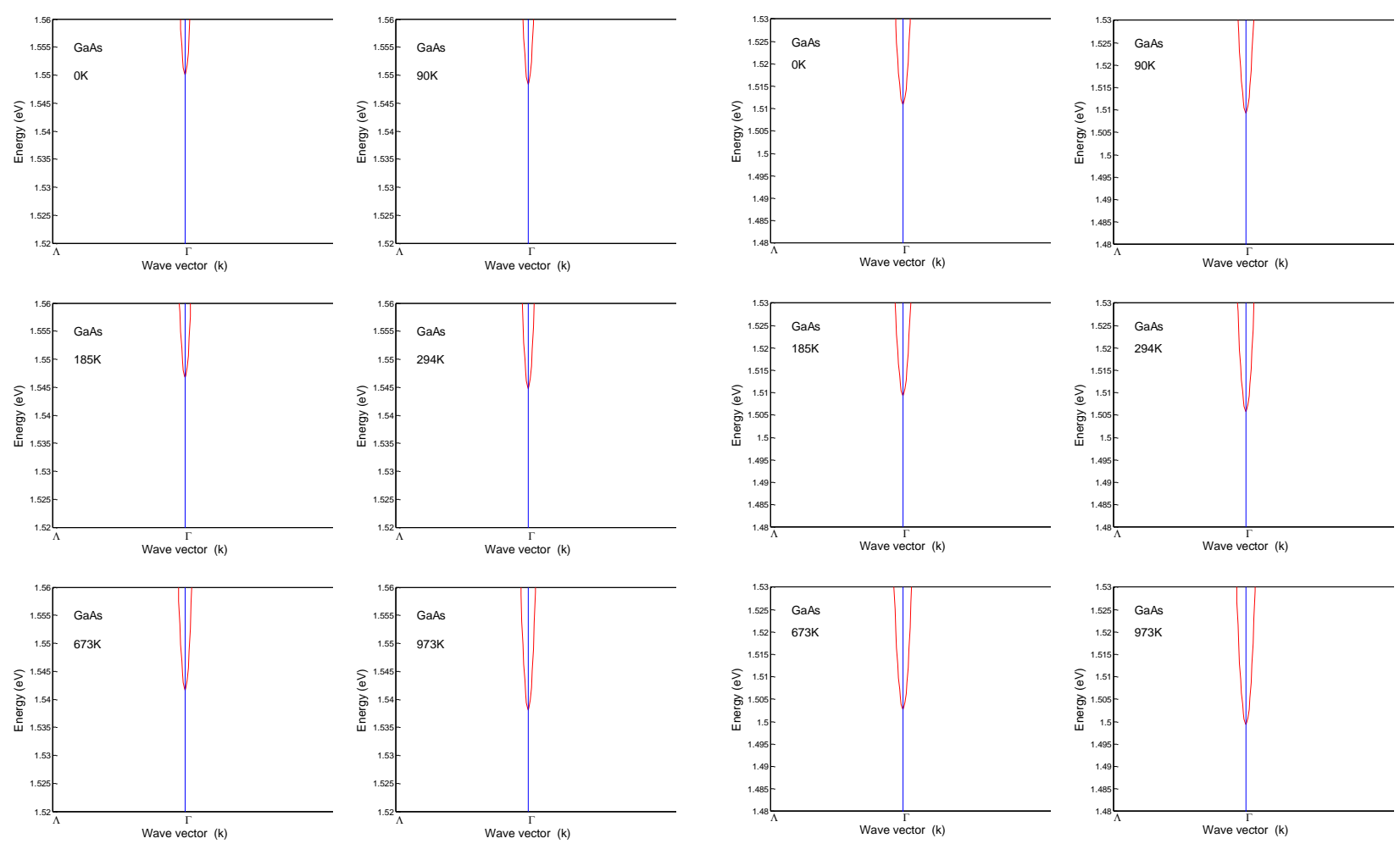

الشكل (8): تركيب حزمة التوصيل عند النقطة م لبلورة GaAs عند

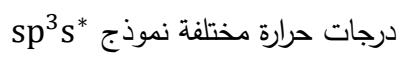

الثكل (7): تركيب حزمة التوصيل عند النقطة م لبلورة GaAs عند

sp sp درجات حرارة مختلفة نموذج
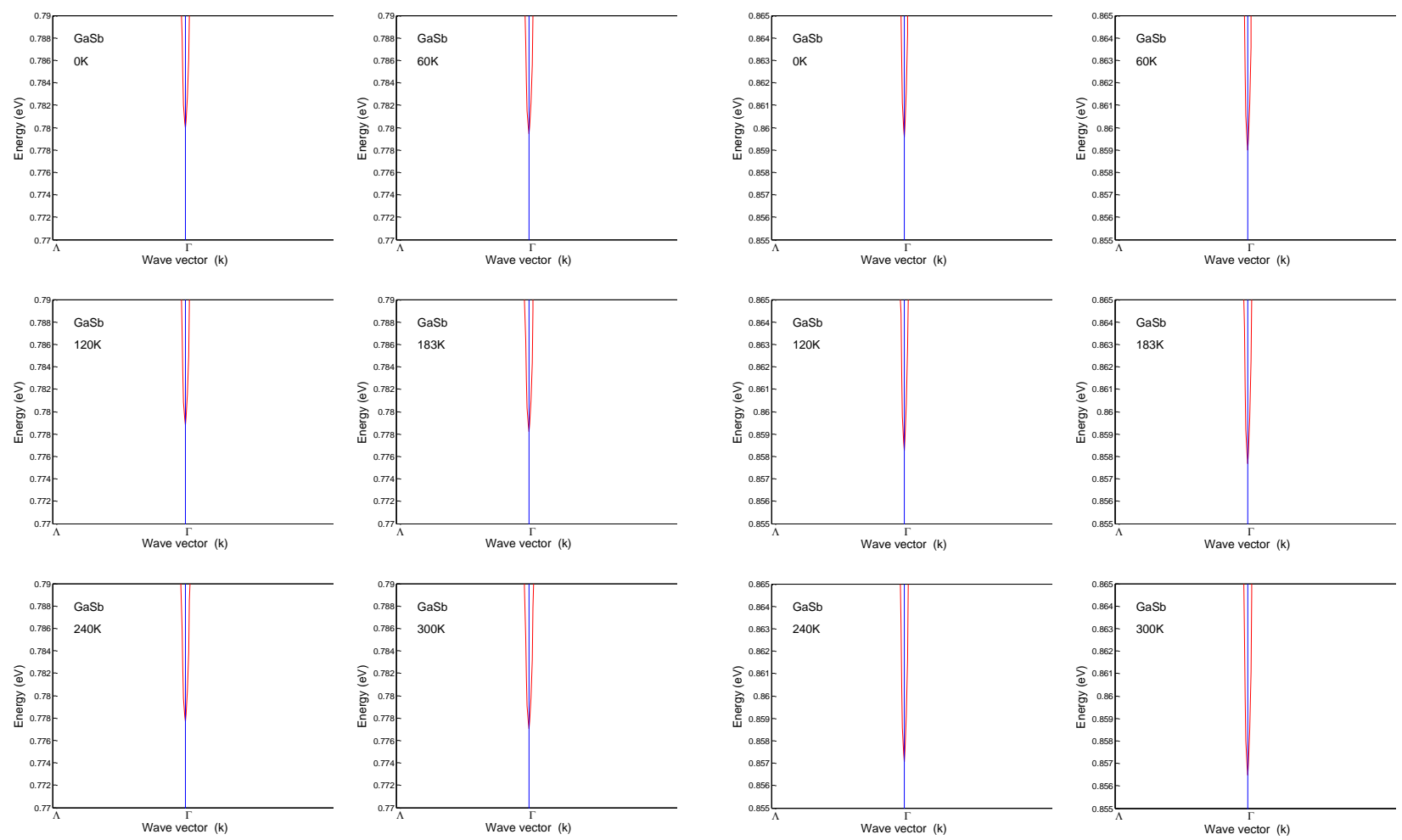

الثكل (10): تركيب حزمة التوصيل عند النقطة م لبلورة GaSb عند

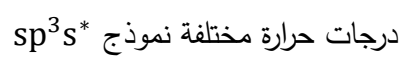
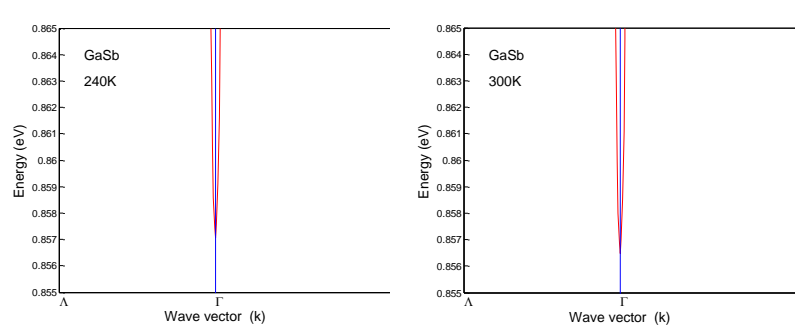

الثكل (9): تركيب حزمة التوصيل عند النقطة م لبلورة GaSb عند sp sp درجات حرارة مختلفة نموذج 

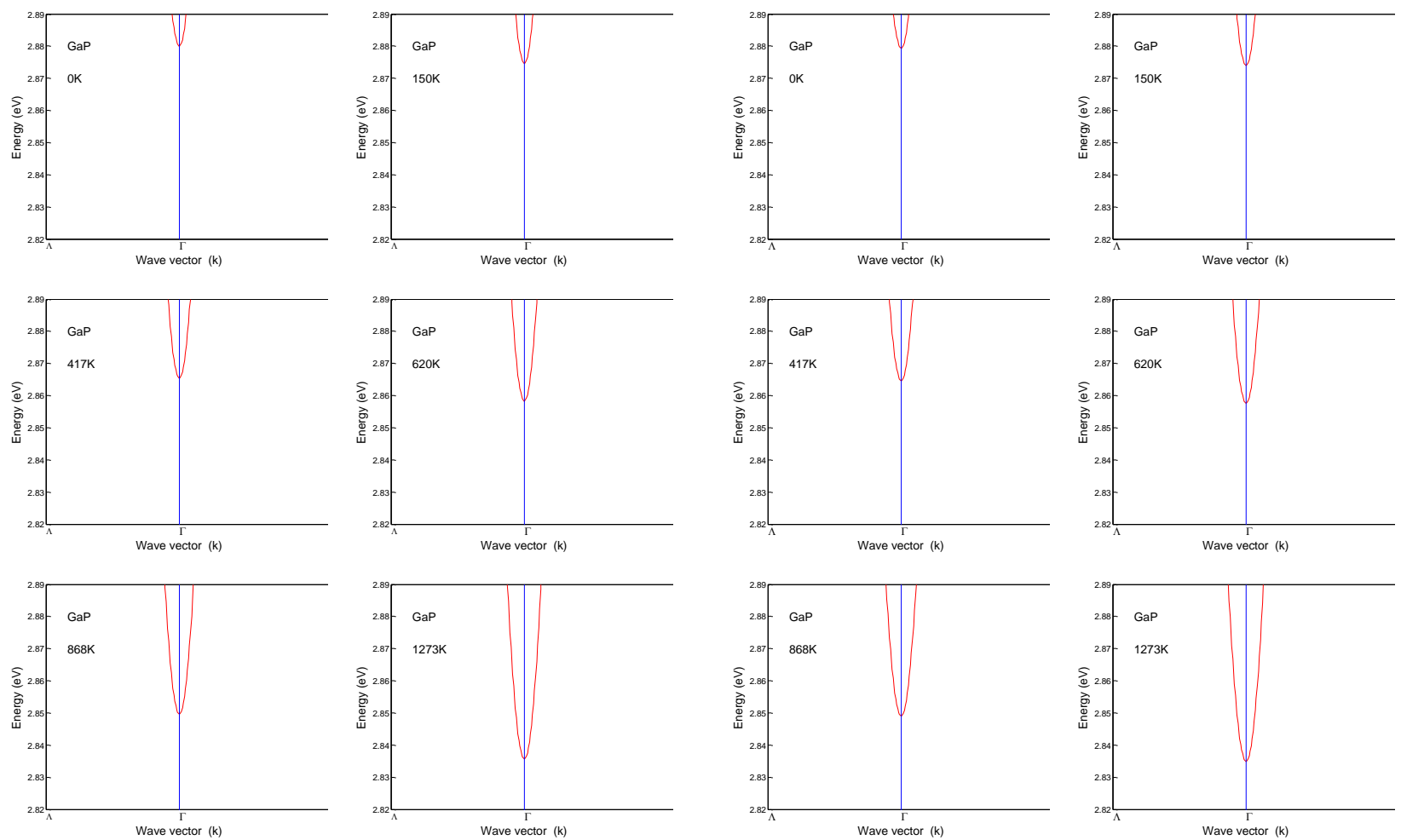

الثكل (12): تركيب حزمة التوصيل عند النقطة م لبلورة GaP عند

الشكل (11): تركيب حزمة التوصيل عند النقطة م لبلورة GaP عند

sp³ s درجات حرارة مختلفة نموذج

$$
\text { sp3 درجات حرارة مختلفة نموذج }
$$

4. تغير فجوة الطاقة للبلورات GaAs، GaSb وGaP مع درجة الحرارة

\section{Variation of energy gap of GaAs, GaSb and GaP crystals with temperature}

يمكن حساب العديد من الخصائص مباشرةً من شكل حزم الطاقة للبلورات GaAs و GaSb GaP عند مدى معين من درجات الحرارة قبل حدوث تغيير في تركيب ZB لهذه البلورات، اذ احتسبت فجوة الطاقة لـ GaAs، GaSb و GaP عند درجات حرارة مختلفة ومباشرةً من حزم الطاقة المحسوبة عند نقاط التماثل العالية L IX و و م كما هو مبين في الجداول (6)، (7) و (8) على التوالي.

الجدول (6): فجوة الطاقة لبلورة GaAs عند نقاط التماثل العالية عند درجات حرارة مختلفة

\begin{tabular}{|c|c|c|c|c|c|c|c|}
\hline \multirow{3}{*}{ Model } & \multirow{2}{*}{$\begin{array}{c}\text { Energy } \\
\text { gap (eV) }\end{array}$} & \multicolumn{6}{|c|}{ Temperature (K) } \\
\cline { 2 - 7 } & $E_{g}^{L}$ & 2.3033 & 2.3009 & 3.2984 & 2.2955 & 2.2856 & 2.2777 \\
\hline \multirow{3}{*}{$\mathrm{sp}^{3}$} & $E_{g}^{X}$ & 4.8808 & 4.8759 & 4.805 & 4.8645 & 4.8434 & 4.8267 \\
\cline { 2 - 8 } & $E_{g}^{K}$ & 4.2272 & 4.2229 & 4.2183 & 4.2130 & 4.1948 & 4.1803 \\
\cline { 2 - 8 } & $E_{g}^{\Gamma}$ & 1.5109 & 1.5094 & 1.50078 & 1.5059 & 1.4994 & 1.4942 \\
\hline \multirow{3}{*}{$\mathrm{sp}^{3} \mathrm{~s}^{*}$} & $E_{g}^{L}$ & 1.6902 & 1.6885 & 1.6867 & 1.6845 & 1.6772 & 1.6715 \\
\cline { 2 - 8 } & $E_{g}^{X}$ & 2.0300 & 2.0279 & 2.0257 & 2.0232 & 2.0144 & 2.0075 \\
\cline { 2 - 8 } & $E_{g}^{K}$ & 2.3516 & 2.3492 & 2.3467 & 2.3437 & 2.3335 & 2.3256 \\
\cline { 2 - 8 } & $E_{g}^{\Gamma}$ & 1.5500 & 1.5483 & 1.5467 & 1.5448 & 1.5380 & 1.5329 \\
\hline
\end{tabular}


Journal of Education and Science (ISSN 1812-125X), Vol: 30, No: 4, 2021 (201-219)

الجدول (7): فجوة الطاقة لبلورة GaSb عند نقاط التماثل العالية عند درجات حرارة مختلفة

\begin{tabular}{|c|c|c|c|c|c|c|c|}
\hline \multirow{3}{*}{ Model } & \multirow{2}{*}{$\begin{array}{c}\text { Energy } \\
\text { gap (eV) }\end{array}$} & \multicolumn{9}{|c|}{ Temperature (K) } \\
\cline { 2 - 8 } & $E_{g}^{L}$ & 2.3503 & 2.3486 & 2.3469 & 2.3451 & 2.3435 & 2.3418 \\
\hline \multirow{3}{*}{$\mathrm{sp}^{3}$} & $E_{g}^{X}$ & 3.6694 & 3.6667 & 3.6640 & 3.6612 & 3.6586 & 3.6560 \\
\cline { 2 - 8 } & $E_{g}^{K}$ & 3.5242 & 3.5216 & 3.5190 & 3.5164 & 3.5139 & 3.5113 \\
\cline { 2 - 9 } & $E_{g}^{\Gamma}$ & 0.8596 & 0.8590 & 0.8583 & 0.8577 & 0.8571 & 0.8565 \\
\hline \multirow{3}{*}{$\mathrm{sp}^{3} \mathrm{~s}^{*}$} & $E_{g}^{L}$ & 0.9621 & 0.9615 & 0.9607 & 0.9600 & 0.9593 & 0.9586 \\
\cline { 2 - 9 } & $E_{g}^{X}$ & 1.2100 & 1.2092 & 1.2082 & 1.2072 & 1.2065 & 1.2056 \\
\cline { 2 - 9 } & $E_{g}^{K}$ & 1.2879 & 1.2870 & 1.2860 & 1.2850 & 1.2841 & 1.2831 \\
\cline { 2 - 9 } & $E_{g}^{\Gamma}$ & 0.7799 & 0.7794 & 0.7788 & 0.7782 & 0.7777 & 0.7770 \\
\hline
\end{tabular}

الجدول (8): فجوة الطاقة لبلورة GaP عند نقاط التماثل العالية عند درجات حرارة مختلفة

\begin{tabular}{|c|c|c|c|c|c|c|c|}
\hline \multirow{3}{*}{ Model } & \multirow{2}{*}{$\begin{array}{c}\text { Energy } \\
\text { gap }(\mathrm{eV})\end{array}$} & \multicolumn{6}{|c|}{ Temperature (K) } \\
\cline { 2 - 8 } & $E_{g}^{L}$ & 3.2685 & 3.2625 & 3.2519 & 3.2439 & 3.2341 & 3.2181 \\
\hline \multirow{3}{*}{$\mathrm{sp}^{3}$} & $E_{g}^{X}$ & 5.4530 & 5.4430 & 5.4253 & 5.4119 & 5.3956 & 5.3689 \\
\cline { 2 - 8 } & $E_{g}^{K}$ & 4.9599 & 4.9508 & 4.9348 & 4.9225 & 4.9077 & 4.8835 \\
\cline { 2 - 8 } & $E_{g}^{\Gamma}$ & 2.8792 & 2.8740 & 2.8646 & 2.8576 & 2.8489 & 2.8349 \\
\hline \multirow{3}{*}{$\mathrm{sp}^{3} \mathrm{~s}^{*}$} & $E_{g}^{L}$ & 2.3968 & 2.3924 & 2.3847 & 2.3788 & 2.3715 & 2.3599 \\
\cline { 2 - 8 } & $E_{g}^{X}$ & 2.3500 & 2.3457 & 2.3381 & 2.3323 & 2.3252 & 2.3138 \\
\cline { 2 - 8 } & $E_{g}^{K}$ & 2.8877 & 2.8824 & 2.8731 & 2.8660 & 2.8572 & 2.8432 \\
\cline { 2 - 8 } & $E_{g}^{\Gamma}$ & 2.8800 & 2.8746 & 2.8654 & 2.8583 & 2.8496 & 2.8356 \\
\hline
\end{tabular}

اقترح Varshni علاقة مهمة جداً لوصف فجوات الطاقة المعتمدة على درجة الحرارة في مختلف بلورات أشباه الموصلات، حيث حقتت هذه العلاقة التجريبية نجاحاً كبيراً في تركيب فجوات الطاقة المعتمدة على درجة الحرارة في العديد من مواد أشباه الموصلات وكذللك في المواد النانوية، تعطى العلاقة التجريبية Varshni's empirical formula لتغيير طاقة فجوة الحزمة مع درجة الحرارة هي [15]:

$$
E_{g}(T)=E_{g}(0)-\frac{A T^{2}}{T+B}
$$

اذ تمثل (T)

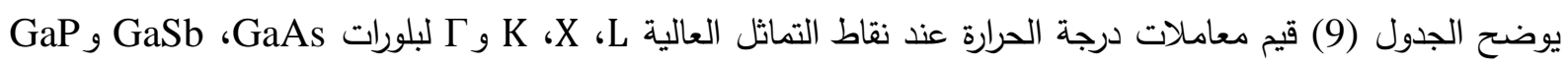


Journal of Education and Science (ISSN 1812-125X), Vol: 30, No: 4, 2021 (201-219)

الجدول (9): معاملات درجة الحرارة للبلورات GaAs و GaSb، GaP

\begin{tabular}{|c|c|c|c|c|c|}
\hline \multirow{2}{*}{ Crystal } & Temperature coefficients & \multicolumn{4}{|c|}{ Symmetry points } \\
\cline { 2 - 6 } & & $\mathrm{L}$ & $\mathrm{X}$ & $\mathrm{K}$ & $\Gamma$ \\
\hline \multirow{3}{*}{$\mathrm{GaAs}$} & $A(\mathrm{~m} \mathrm{eV} / \mathrm{K})$ & 0.605 & 0.460 & 0.5405 & 0.5405 \\
\cline { 2 - 7 } & $B(\mathrm{~K})$ & 204 & 204 & 204 & 204 \\
\hline \multirow{2}{*}{$\mathrm{GaSb}$} & $A(\mathrm{meV} / \mathrm{K})$ & 0.597 & 0.475 & 0.475 & 0.417 \\
\cline { 2 - 7 } & $B(\mathrm{~K})$ & 140 & 94 & 94 & 140 \\
\hline \multirow{2}{*}{$\mathrm{GaP}$} & $A(\mathrm{meV} / \mathrm{K})$ & 0.5771 & 0.5771 & 0.5771 & 0.5771 \\
\cline { 2 - 6 } & $B(\mathrm{~K})$ & 372 & 372 & 372 & 372 \\
\hline
\end{tabular}

Ref. [13]

تم استخدام معادلة Varshni لإيجاد فجوة الطاقة لـ GaSb، GaPs و عند نقاط التماثل العالية L، X، K و م وكما مبين في الجداول (10)، (11) و (12) على التوالي.

الجدول (10): تغير فجوة الطاقة مع درجة الحرارة عند نقاط التماثل العالية باستخدام معادلة Varshni لبلورة GaAs

\begin{tabular}{|c|c|c|c|c|c|c|c|}
\hline \multirow{3}{*}{ Model } & \multirow{2}{*}{$\begin{array}{c}\text { Energy } \\
\text { gap }(\mathrm{eV})\end{array}$} & \multicolumn{9}{|c|}{ Temperature (K) } \\
\cline { 2 - 8 } & $E_{g}^{L}$ & 2.3033 & 2.2866 & 2.2501 & 2.1983 & 1.9908 & 1.8167 \\
\hline \multirow{3}{*}{$\mathrm{sp}^{3}$} & $E_{g}^{X}$ & 4.8808 & 4.8681 & 4.8403 & 4.8010 & 4.6432 & 4.5108 \\
\cline { 2 - 8 } & $E_{g}^{K}$ & 4.2272 & 4.2123 & 4.1796 & 4.1334 & 3.9481 & 3.7924 \\
\cline { 2 - 9 } & $E_{g}^{\Gamma}$ & 1.5109 & 1.4960 & 1.4633 & 1.4171 & 1.2318 & 1.0761 \\
\hline \multirow{3}{*}{$\mathrm{sp}^{3} \mathrm{~s}^{*}$} & $E_{g}^{L}$ & 1.6902 & 1.6735 & 1.6370 & 1.5852 & 1.3777 & 1.1152 \\
\cline { 2 - 8 } & $E_{g}^{X}$ & 2.0300 & 2.0173 & 1.9895 & 1.9502 & 1.7924 & 1.6600 \\
\cline { 2 - 8 } & $E_{g}^{K}$ & 2.3516 & 2.3367 & 2.3040 & 2.2578 & 2.0725 & 1.9168 \\
\cline { 2 - 8 } & $E_{g}^{\Gamma}$ & 1.5500 & 1.5351 & 1.5024 & 1.4562 & 1.2709 & 1.1152 \\
\hline
\end{tabular}

الجدول (11): تغير فجوة الطاقة مع درجة الحرارة عند نقاط التماثل العالية باستخدام معادلة Varshni لبلورة GaSb

\begin{tabular}{|c|c|c|c|c|c|c|c|}
\hline \multirow{3}{*}{ Model } & \multirow{2}{*}{$\begin{array}{c}\text { Energy } \\
\text { gap (eV) }\end{array}$} & \multicolumn{7}{|c|}{ Temperature (K) } \\
\cline { 2 - 8 } & $E_{g}^{L}$ & 2.3503 & 2.3396 & 2.3172 & 2.2884 & 2.2598 & 2.2282 \\
\hline \multirow{3}{*}{$\mathrm{sp}^{3}$} & $E_{g}^{X}$ & 3.6694 & 3.6583 & 3.6374 & 3.6120 & 3.5875 & 3.5609 \\
\cline { 2 - 8 } & $E_{g}^{K}$ & 3.5242 & 3.5131 & 3.4922 & 3.4668 & 3.4423 & 3.4157 \\
\cline { 2 - 8 } & $E_{g}^{\Gamma}$ & 0.8596 & 0.8521 & 0.8365 & 0.8164 & 0.7964 & 0.7743 \\
\hline \multirow{3}{*}{$\mathrm{sp}^{3} \mathrm{~s}^{*}$} & $E_{g}^{L}$ & 0.9621 & 0.9514 & 0.9290 & 0.9002 & 0.8716 & 0.8400 \\
\cline { 2 - 8 } & $E_{g}^{X}$ & 1.2100 & 1.1989 & 1.1780 & 1.1526 & 1.1281 & 1.1015 \\
\cline { 2 - 8 } & $E_{g}^{K}$ & 1.2879 & 1.2768 & 1.2559 & 1.2305 & 1.2060 & 1.1794 \\
\cline { 2 - 8 } & $E_{g}^{\Gamma}$ & 0.7799 & 0.7724 & 0.7568 & 0.7367 & 0.7167 & 0.6946 \\
\hline
\end{tabular}


Journal of Education and Science (ISSN 1812-125X), Vol: 30, No: 4, 2021 (201-219)

الجدول (12): تغير فجوة الطاقة مع درجة الحرارة عند نقاط التماثل العالية باستخدام معادلة Varshni لبلورة GaP

\begin{tabular}{|c|c|c|c|c|c|c|c|}
\hline \multirow{3}{*}{ Model } & \multirow{2}{*}{$\begin{array}{c}\text { Energy } \\
\text { gap }(\mathrm{eV})\end{array}$} & \multicolumn{9}{|c|}{ Temperature (K) } \\
\cline { 2 - 8 } & $E_{g}^{L}$ & 3.2685 & 3.2436 & 3.1413 & 3.0449 & 2.9179 & 2.7000 \\
\hline \multirow{3}{*}{$\mathrm{sp}^{3}$} & $E_{g}^{X}$ & 5.4530 & 5.4281 & 5.3258 & 5.2294 & 5.1024 & 4.8845 \\
\cline { 2 - 8 } & $E_{g}^{K}$ & 4.9599 & 4.9350 & 4.8327 & 4.7363 & 4.6093 & 4.3914 \\
\cline { 2 - 8 } & $E_{g}^{\Gamma}$ & 2.8792 & 2.8543 & 2.7520 & 2.6556 & 2.5286 & 2.3107 \\
\hline \multirow{3}{*}{$\mathrm{sp}^{3} \mathrm{~s}^{*}$} & $E_{g}^{L}$ & 2.3968 & 2.3719 & 2.2696 & 2.1732 & 2.0462 & 1.8283 \\
\cline { 2 - 8 } & $E_{g}^{X}$ & 2.3500 & 2.3251 & 2.2228 & 2.1264 & 1.9994 & 1.7815 \\
\cline { 2 - 8 } & $E_{g}^{K}$ & 2.8877 & 2.8628 & 2.7605 & 2.6641 & 2.5371 & 2.3192 \\
\cline { 2 - 8 } & $E_{g}^{\Gamma}$ & 2.8800 & 2.8551 & 2.7528 & 2.6564 & 2.5294 & 2.3115 \\
\hline
\end{tabular}

توضح الاشكال (13)، (14)، (15)، (16)، (17) و(18) كيفية تغير فجوة الطاقة مع تغير درجة الحرارة لـ GaAs،

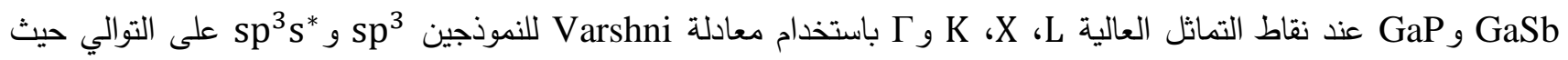
نلاحظ انخفاض في قيمة فجوة الطاقة مع زيادة درجات الحرارة. هناك طريقتين لتفسير تأثير درجة الحرارة على فجوة الطاقة لأشباه الموصلات، الطريقة الاولى هي انه عندما ترتفع درجة الحرارة فأن سعة اهتزاز الذرات سوف تزداد مما يؤدي الى كبر المسافة بين الذرات، وبما ان فجوة الطاقة تتاسب بشكل عكسي مع مربع ثابت الثبيكة لذا فإن زيادة ثابت الثبيكة يؤدي الى التمدد الحراري للثبيكة وتقليل فجوة الطاقة. اما الطريقة الثانية فهي نتيجة التفاعلات التي تحدث بين الإكترون والفونون مما يؤدي الى اضطراب في فجوة الطاقة، حيث تكتسب الاككترونات طاقة حرارية وتتحول الى ادنى مستويات طاقية ممكنة قبل ان تندمج مع الفجوات وبذلك تظهر اقل فجوة طاقة [16].

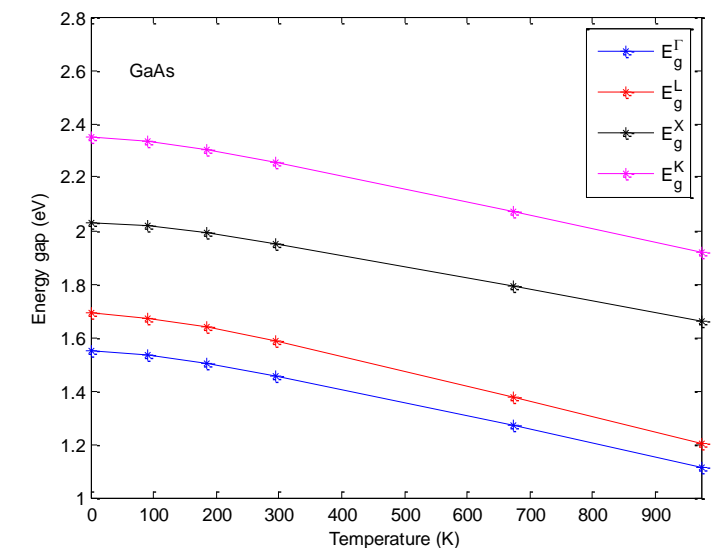

الثكل (14): تغير فجوة الطاقة مع درجة الحرارة عند نقاط التماثل العالية

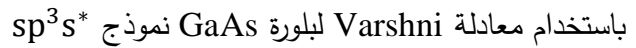

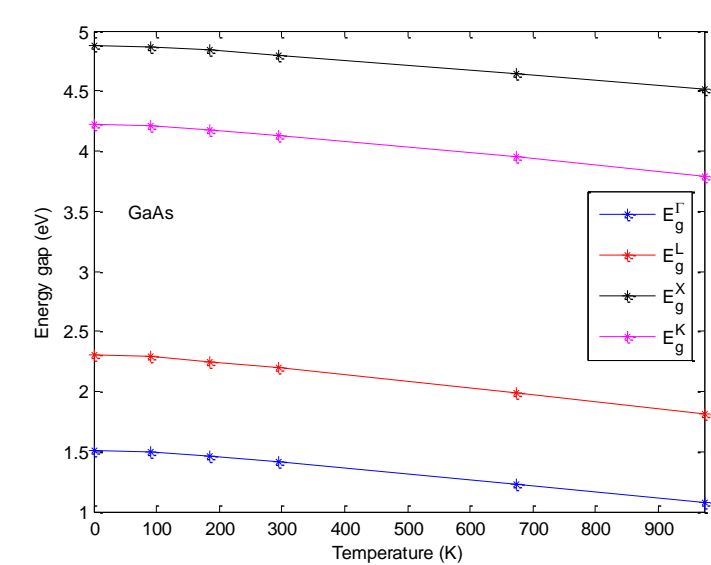

الثكل (13): تغير فجوة الطاقة مع درجة الحرارة عند نقاط التماثل العالية باستخدام معادلة Varshni لبلورة GaAs نموذج 


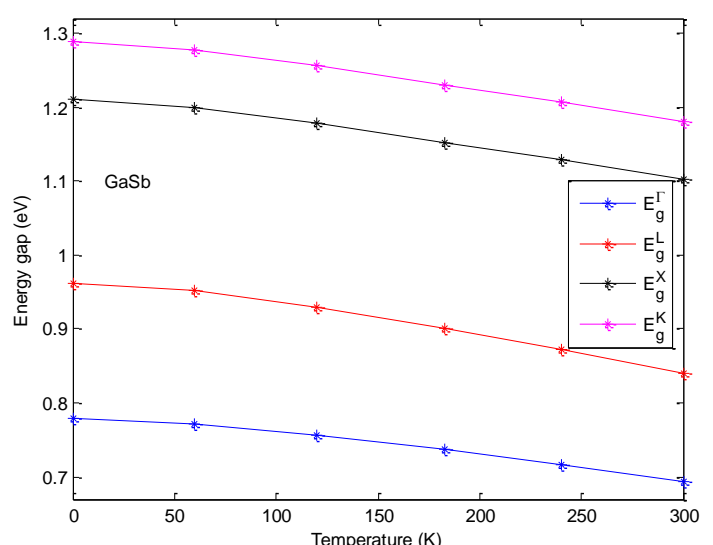

الشكل (16): تغير فجوة الطاقة مع درجة الحرارة عند نقاط التماثل العالية باستخدام معادلة Garshni لبلورة GaSb نموذج

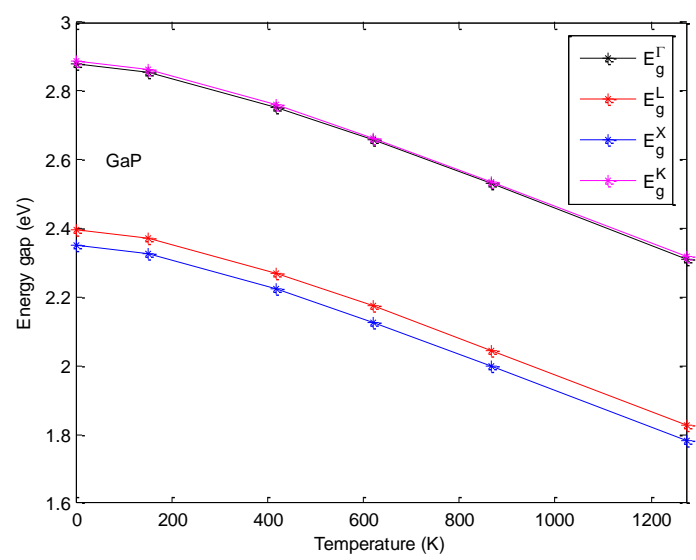

الثكل (18): تغير فجوة الطاقة مع درجة الحرارة عند نقاط التماثل العالية sp باستخدام معادلة Varshni لبلورة GaP نموذج

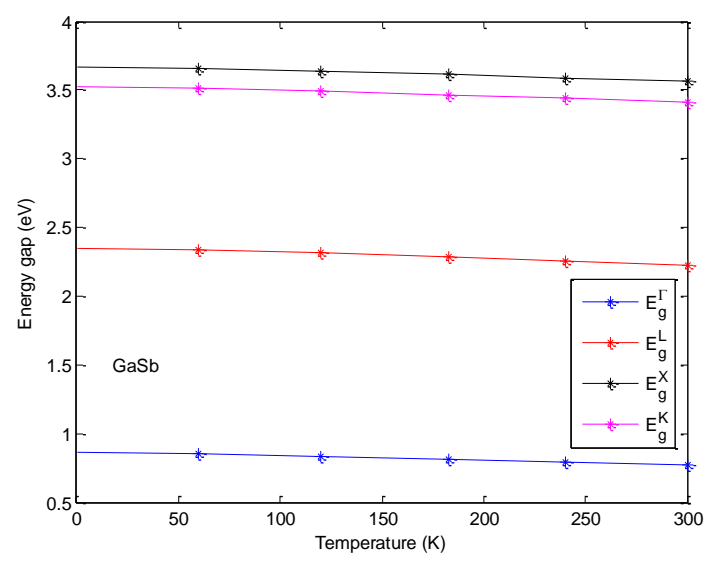

الثكل (15): تغير فجوة الطاقة مع درجة الحرارة عند نقاط التماثل العالية باستخدام معادلة Varshni لبلورة GaSb

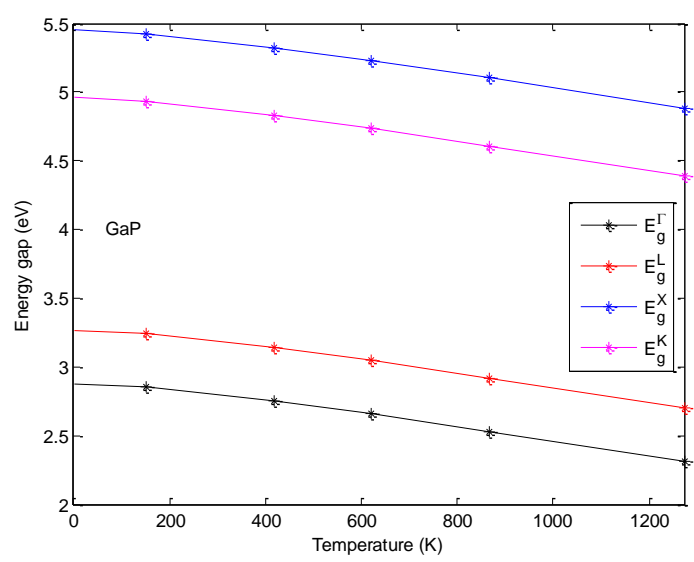

الثكل (17): تغير فجوة الطاقة مع درجة الحرارة عند نقاط التماثل العالية باستخدام معادلة Varshni لبلورة GaP نموذج

بعد استخدام معادلة Varshni وايجاد فجوة الطاقة للبلورات GaA و GaSb GaA كدالة لدرجة الحرارة عند نقاط التماثل

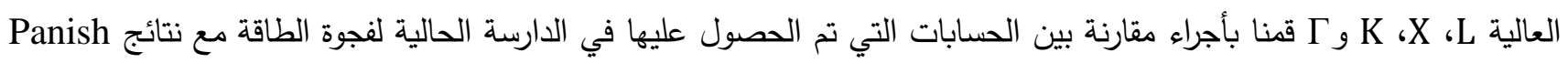

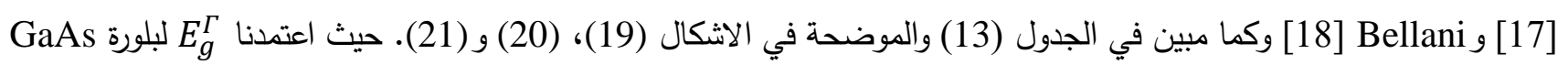

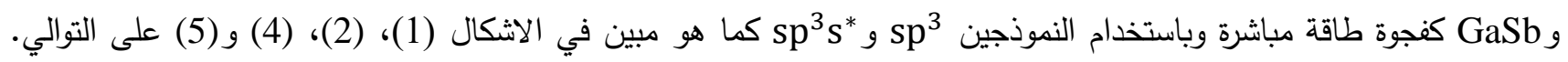

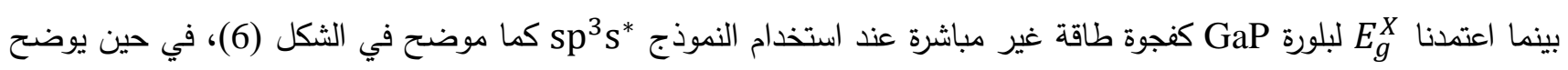
الثكل (3) بأن اقل قيمة لفجوة الطاقة لبلورة GaP تكون عند النقطة م عند استخدام النموذج sp لان هذا النموذج لا يعطي وصف دقيق لحزمة التوصيل. حيث اظهرت النتائج ان نموذج sp³ يعطي نتائج افضل من النموذج sp وذلك نتيجة لزيادة حجم مصفوفة الهاميلتون H. 
Journal of Education and Science (ISSN 1812-125X), Vol: 30, No: 4, 2021 (201-219)

الجدول (13): تغير فجوة الطاقة مع درجة الحرارة عند بعض نقاط التماثل العالية باستخدام معادلة Varshni للبلورات GaP GaSb،GaAs و

\begin{tabular}{|c|c|c|c|c|c|c|c|c|c|}
\hline \multirow{2}{*}{ Crystal } & \multirow{2}{*}{$\begin{array}{c}\text { Energy } \\
\text { gap }(\mathrm{eV})\end{array}$} & \multirow{2}{*}{ Model } & \multicolumn{6}{|c|}{ Temperature $(\mathrm{K})$} & \multirow{2}{*}{ Ref. } \\
\hline & & & 0 & 90 & 185 & 294 & 673 & 973 & \\
\hline \multirow{3}{*}{ GaAs } & \multirow{3}{*}{$E_{g}^{\Gamma}$} & $\mathrm{sp}^{3}$ & 1.5109 & 1.4960 & 1.4633 & 1.4171 & 1.2318 & 1.2318 & This work \\
\hline & & $\mathrm{sp}^{3} \mathrm{~s}^{*}$ & 1.5500 & 1.5351 & 1.5024 & 1.4562 & 1.2709 & 1.1152 & This work \\
\hline & & & 1.522 & 1.510 & 1.481 & 1.437 & 1.252 & 1.091 & [17] \\
\hline \multirow{2}{*}{ Crystal } & \multirow{2}{*}{$\begin{array}{c}\text { Energy } \\
\text { gap }(e V)\end{array}$} & \multirow{2}{*}{ Model } & \multicolumn{6}{|c|}{ Temperature (K) } & \multirow{2}{*}{ Ref. } \\
\hline & & & 0 & 60 & 120 & 183 & 240 & 300 & \\
\hline \multirow{3}{*}{$\mathrm{GaSb}$} & \multirow{3}{*}{$E_{g}^{\Gamma}$} & $\mathrm{sp}^{3}$ & 0.8596 & 0.8521 & 0.8365 & 0.8164 & 0.7964 & 0.7743 & This work \\
\hline & & $\mathrm{sp}^{3} \mathrm{~s}^{*}$ & 0.7799 & 0.7724 & 0.7568 & 0.7367 & 0.7167 & 0.6946 & This work \\
\hline & & & 0.81 & 0.805 & 0.791 & 0.771 & 0.748 & 0.723 & [18] \\
\hline \multirow{2}{*}{ Crystal } & \multirow{2}{*}{$\begin{array}{c}\text { Energy } \\
\text { gap }(\mathrm{eV})\end{array}$} & \multirow{2}{*}{ Model } & \multicolumn{6}{|c|}{ Temperature $(\mathrm{K})$} & \multirow{2}{*}{ Ref. } \\
\hline & & & 0 & 150 & 417 & 620 & 868 & 1273 & \\
\hline \multirow{3}{*}{$\mathrm{GaP}$} & $E_{g}^{\Gamma}$ & $\mathrm{sp}^{3}$ & 2.8792 & 2.8543 & 2.7520 & 2.6556 & 2.5286 & 2.3107 & This work \\
\hline & $E_{g}^{X}$ & $\mathrm{sp}^{3} \mathrm{~s}^{*}$ & 2.3500 & 2.3251 & 2.2228 & 2.1264 & 1.9994 & 1.7815 & This work \\
\hline & & & 2.338 & 2.315 & 2.215 & 2.117 & 1.986 & 1.758 & [17] \\
\hline
\end{tabular}

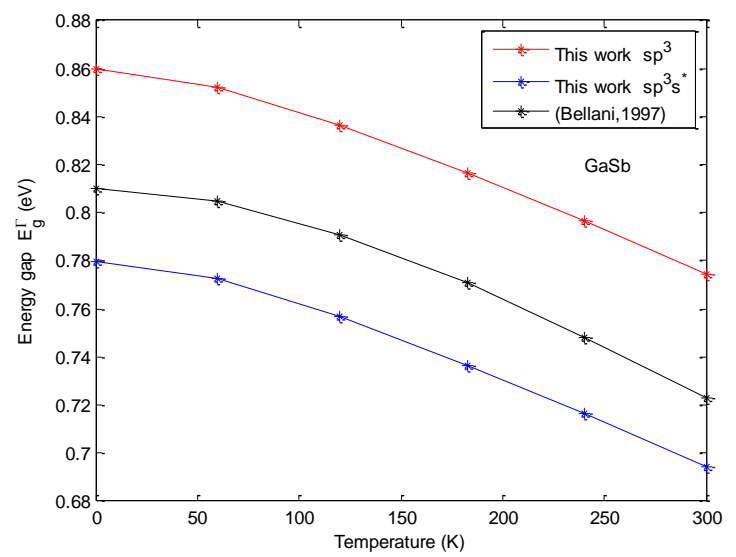

الثكل (20): تغير فجوة الطاقة مع درجة الحرارة عند نقطة التماثل م

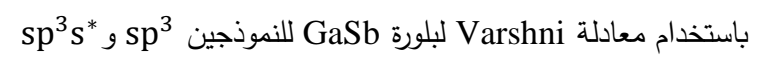
ومقارنتها مع [18]

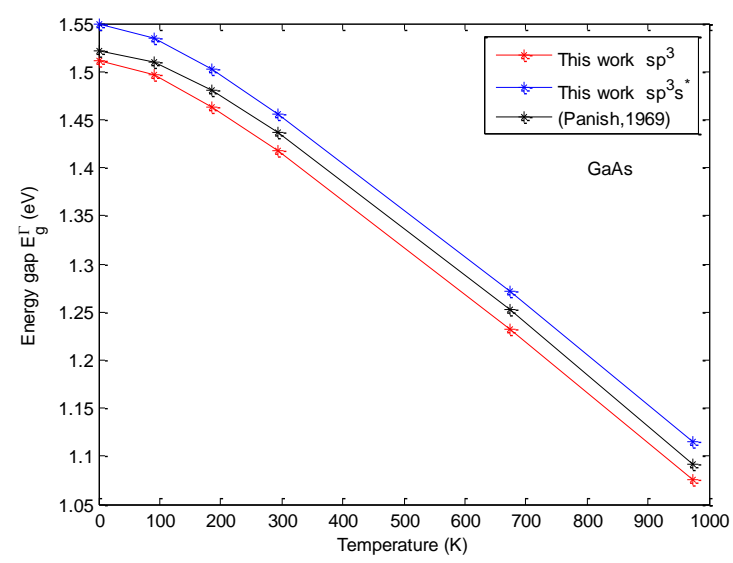

الثكل (19): تغير فجوة الطاقة مع درجة الحرارة عند نقطة التماثل م

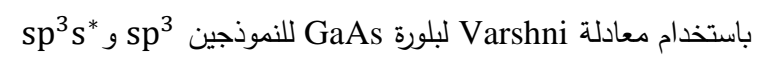

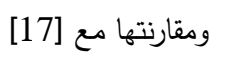




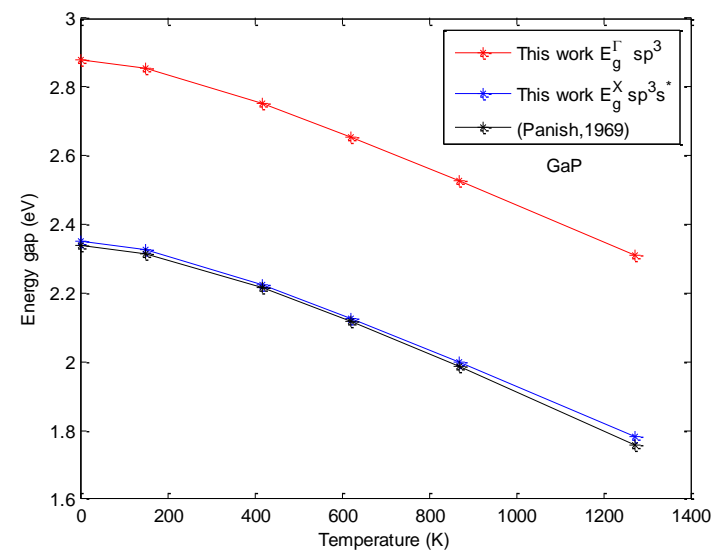

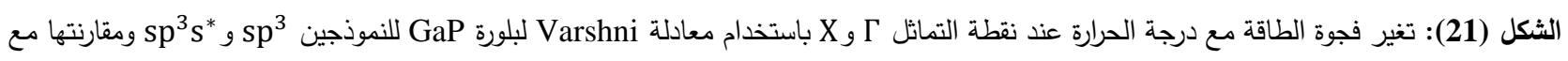

[17]

5. تغير معامل الانكسار للبلورات GaAs، GaSb وGaP مع درجة الحرارة Variation of refractive index of GaAs, GaSb and GaP crystals with temperature

يتغير معامل الانكسار للبلورات GaP و GaSb GaAs مع تغير درجة الحرارة ولمدى درجات الحرارة المستخدمة في هذه

الدراسة, اذ تم استخدام علاقة Moss لإيجاد معامل الانكسار بالنسبة لهذه البلورات عند درجات حرارة مختلفة وكما موضح في الجدول

(14) وحسب الصيغة الاتية [19]:

$$
\mathrm{n}^{4}=\frac{95}{E_{g}^{\Gamma}}
$$

اذ نلاحظ بان معامل الانكسار سوف يزداد بشكل تدريجي مع زيادة درجات الحرارة وكما موضح في الاشكال (22)، (23) و(24) لبلورات GaAs، GaSb و GaP على التوالي. الجدول (14): قيمة معامل الانكسار لبلورات GaAs، GaSb GaPs باستخدام صيغة Mosa عند درجات حرارة مختلفة

\begin{tabular}{|c|c|c|c|c|c|c|c|c|c|}
\hline \multirow{2}{*}{ Crystal } & \multirow{2}{*}{$\begin{array}{l}\text { Refractive } \\
\text { Index }\end{array}$} & \multirow{2}{*}{ Model } & \multicolumn{6}{|c|}{ Temperature (K) } & \multirow{2}{*}{ Ref. } \\
\hline & & & 0 & 90 & 185 & 294 & 673 & 973 & \\
\hline \multirow{2}{*}{ GaAs } & \multirow{2}{*}{$\mathrm{n}$} & $\mathrm{sp}^{3}$ & 2.8159 & 2.8166 & 2.8174 & 2.8183 & 2.8213 & 2.8238 & \multirow{2}{*}{$\begin{array}{l}3.30[19] \\
2.85[20]\end{array}$} \\
\hline & & $\mathrm{sp}^{3} \mathrm{~s}^{*}$ & 2.7980 & 2.7988 & 2.7995 & 2.8004 & 2.8034 & 2.8058 & \\
\hline \multirow{2}{*}{ Crystal } & \multirow{2}{*}{$\begin{array}{l}\text { Refractive } \\
\text { Index }\end{array}$} & \multirow{2}{*}{ Model } & \multicolumn{6}{|c|}{ Temperature $(\mathrm{K})$} & \multirow{2}{*}{ Ref. } \\
\hline & & & 0 & 60 & 120 & 183 & 240 & 300 & \\
\hline \multirow{2}{*}{$\mathrm{GaSb}$} & \multirow{2}{*}{$\mathrm{n}$} & $\mathrm{sp}^{3}$ & 3.2423 & 3.2429 & 3.2436 & 3.2441 & 3.2447 & 3.2453 & \multirow{2}{*}{$\begin{array}{l}3.79[19] \\
3.29[20] \\
\end{array}$} \\
\hline & & $\mathrm{sp}^{3} \mathrm{~s}^{*}$ & 3.3222 & 3.3227 & 3.3233 & 3.3240 & 3.3245 & 3.3253 & \\
\hline \multirow{2}{*}{ Crystal } & \multirow{2}{*}{$\begin{array}{l}\text { Refractive } \\
\text { Index }\end{array}$} & \multirow{2}{*}{ Model } & \multicolumn{6}{|c|}{ Temperature $(\mathrm{K})$} & \multirow{2}{*}{ Ref. } \\
\hline & & & 0 & 150 & 417 & 620 & 868 & 1273 & \\
\hline \multirow{2}{*}{$\mathrm{GaP}$} & \multirow{2}{*}{$\mathrm{n}$} & $\mathrm{sp}^{3}$ & 2.3967 & 2.3978 & 2.3997 & 2.4012 & 2.4030 & 2.4060 & \multirow{2}{*}{$\begin{array}{l}2.90[19] \\
2.55[20]\end{array}$} \\
\hline & & $\mathrm{sp}^{3} \mathrm{~s}^{*}$ & 2.3965 & 2.3977 & 2.3996 & 2.4011 & 2.4029 & 2.4059 & \\
\hline
\end{tabular}




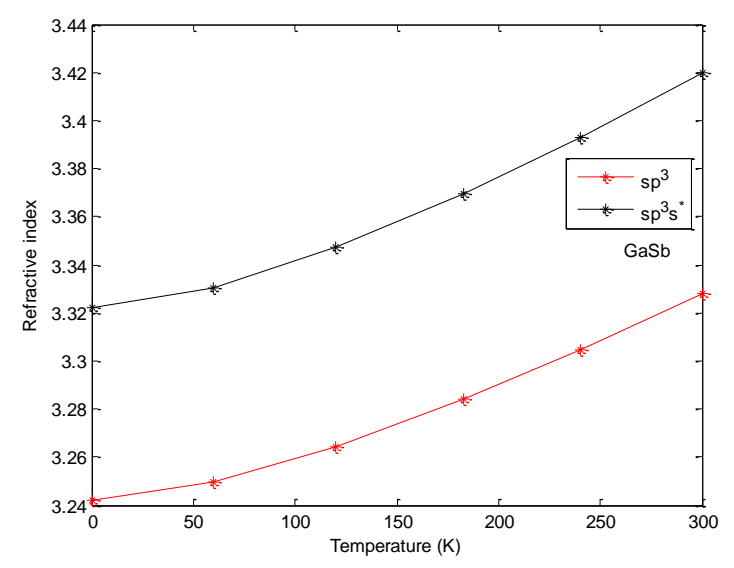

الثكل (23): تغير معامل الانكسار مع درجة الحرارة عند نقطة التماثل م باستخدام صيغة Moss لبلورة GaSb للنموذجين sps

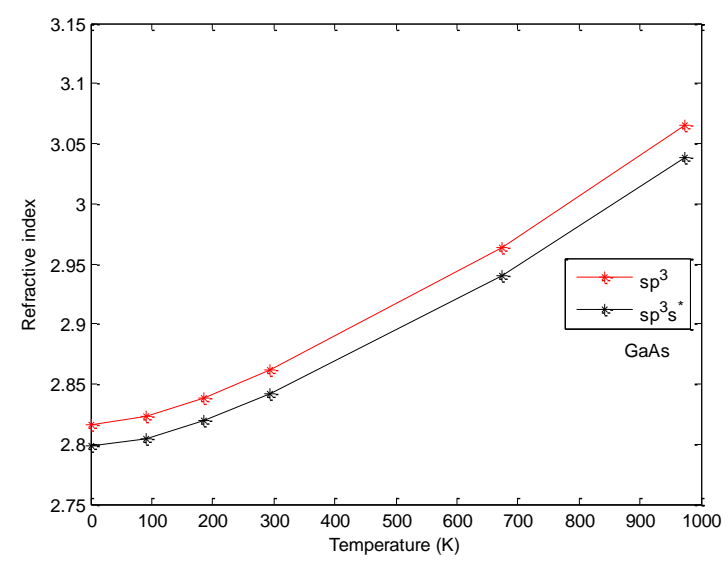

الثكل (22): تغير معامل الانكسار مع درجة الحرارة عند نقطة التماثل م

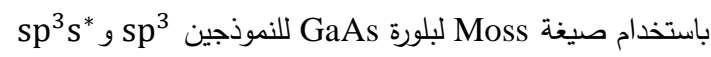

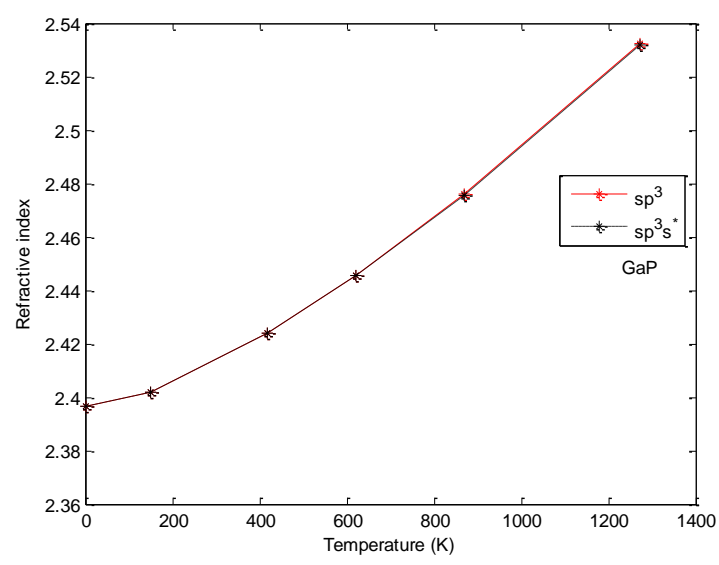

الشكل (24): تغير معامل الانكسار مع درجة الحرارة عند نقطة التماثل م باستخدام صيغة Moss لبلورة GaP للنموذجين sp و وs

6. المناقشة والاستنتاجات

تم حساب تركيب حزم الطاقة الأكترونية في مواد اشباه الموصلات المركبة III-V للبلورات GaAs، GaSb و GaP عند درجات حرارة مختلفة باستخدام طريقة الربط المحكم وللنموذجين sp وs sp3، اذ تعتبر هذه الطريقة من الطرائق التجريبية الدقيقة والمناسبة لحساب تركيب حزم الطاقة نظراً لانخفاض التكاليف الحسابية، وقد بينت النتائج من خلال هذا البرنامج تطابقاً جيداً مع البحوث المنشورة ومن خلال النتائج تم استتتاج ما يأتي:

عند حساب تركيب الحزم الاككترونية للبلورات GaAs، GaP و GaSb ذات تركيب ZB تحت تأثير درجة الحرارة، لاحظنا وجود ازاحة طفيفة في حزم التوصيل نحو الاسفل في الاشكال (7)، (8)، (9)، (10)، (11) و(12). وبذلك نحصل على انخفاض في قيم فجوة الطاقة مع زيادة درجة الحرارة. عند حساب تأثير درجة الحرارة للبلورات GaAs و GaSb GaP وجدنا ان فجوة الطاقة تقل مع ارتفاع درجة الحرارة، وهذا يتوافق مع النمط العام لسلوك تغير فجوة الطاقة مع درجة الحرارة لأشباه الموصلات. عند استخدام معادلة Varshni لإيجاد فجوة الطاقة للبلورات GaP,GaSb GaAs، عند نقاط التماثل العالية L، X K و م لاحظنا وجود توافق بين 
الحسابات التي تم الحصول عليها في الدارسة الحالية لفجوة الطاقة عند النقطة م لبلورة GaAs و GaSb مع نتائج Panish [17 عند استخدام كلا النموذجين، في حين ان نتائج فجوة الطاقة لبلورة GaP عند النقطة استخدام النموذج sps عكون اكثر توافقاً مع نتائج Bellani [18] من النقطة م عند استخدام النموذج sp3، وذلك لان بلورة GaP تمتلك فجوة طاقة غير مباشرة في حين أن البلورتين GaSb GaAs فإننا لا نلاحظ هذا الاختلاف عند استخدام النموذجين وذلك لانهما يمتلكان فجوة طاقة مباشرة. من خلال نتائج تركيب حزم الطاقة مع درجة الحرارة تم حساب تغير معامل الانكسار n مع درجة الحرارة باستخدام صيغة Moss حيث بينت النتائج التي تم الحصول عليها زيادة معامل الانكسار مع ارتفاع درجة الحرارة. 7. يتقدم الباحثان بالشكر والتقدير الى جامعة الموصل وكلية التربية للعلوم الصرفة وقسم الفيزياء على دعم البحث.

[1] O.L. Anderson, H. Oda and D. Isaak, "A model for the computation of thermal expansivity at high compression and high temperatures: $\mathrm{MgO}$ as an example", J. Geophysical Research Letters, 19 (19): pp. 1987-1990, 1992. https://doi.org/10.1029/92GL02145

[2] P. Geng, W. Li, X. Zhang, X. Zhang, Y. Deng and H. Kou, "A novel theoretical model for the temperature dependence of band gap energy in semiconductors", J. Applied. Phys., 50 (40): pp. 40LT02, 2017.

[3] T. Ismail and M. Hussien, "Band Structure of GaAs Crystal Using the Semiempirical Tight Binding Method", appear in Journal of Education and Science, 30(3), 2021.

[4] A. Di Carlo, "Microscopic theory of nanostructured semiconductor devices: beyond the envelopefunction approximation", J. Semiconductor Science and Technology, 18 (1): pp. R1. 2002.

[5] D. Chadi, "Spin-orbit splitting in crystalline and compositionally disordered semiconductors", J. Phys. Rev. B., 16 (2): pp. 790-796, 1977. https://doi.org/10.1103/PhysRevB.16.790

[6] P. Vogl, H. P. Hjalmarson and J. D. Dow, "A semi-empirical tight-binding theory of the electronic structure of semiconductors", J. Phys. Chem. Sol., 44 (5): pp. 365-378, 1983. https://doi.org/10.1016/0022-3697(83)90064-1

[7] G. Gopir, N. Zulkifli, and A. Othman, "Electronic Structure Calculation of Bulk Semiconductors Using The $\mathrm{Sp}^{3} \mathrm{~s}^{*}$ Empirical Tight Binding Method", Solid State Science and Technology, vol. 13, no. 1 \& 2, pp.234-243, 2005.

[8] W.A. Harrison, Electronic structure and the properties of solids: the physics of the chemical bond. Courier Corporation, 2012.

[9] E. Pierron, D. Parker and J. McNeely, "Coefficient of expansion of gallium arsenide from - 62 to 200 C", J. Acta Crystallographica, 21 (2): pp. 290-290, 1966. https://doi.org/10.1107/S0365110X66002780

[10] H. Detz, "Thermal expansion of III-V materials in atomistic models using empirical Tersoff potentials", J. Electronics Letters, 51 (18): pp. 1455-1457, 2015.

[11] R. Indolia, "To Study the dependency of linear thermal expansion coefficient and Melting Temperature on Plasmon energy in case of II-VI and III-V groups of binary semiconductors", J. International Journal and Applied Physics, 13 (3): pp. 451-457, 2017.

[12] K.Y. Cheng, III-V Compound Semiconductors and Devices: An Introduction to Fundamentals. Springer Nature, 2020. 
[13] I. Vurgaftman, J.áR. Meyer and L.áR. Ram-Mohan, "Band parameters for III-V compound semiconductors and their alloys", J. Applied. Phys., 89 (11): pp. 5815-5875, 2001. https://doi.org/10.1063/1.1368156

[14] Y. Sun, S. E. Thompson and T. Nishida, Strain effect in semiconductors: theory and device applications. Springer Science \& Business Media, 2009.

[15] C.-C. Li, M. Gong, X.-D. Chen, S. Li, B.-W. Zhao, Y. Dong, G.-C. Guo and F.-W. Sun, "Temperature dependent energy gap shifts of single color center in diamond based on modified Varshni equation", J. Diamond and Related Materials, 74: pp. 119-124, 2017. https://doi.org/10.1016/j.diamond.2017.03.002

[16] Y.P. Varshni, Temperature dependence of the energy gap in semiconductors, J. physica, 34 (1): pp. 149-154, 1967. https://doi.org/10.1016/0031-8914(67)90062-6

[17] M. Panish and Jr. H. Casey, "Temperature dependence of the energy gap in GaAs and GaP", J. Applied. Phys., 40 (1): pp. 163-167, 1969. https://doi.org/10.1063/1.1657024

[18] V. Bellani, S. Di Lernia, M. Geddo, G. Guizzetti, A. Bosacchi, S. Franchi and R. Magnanini, Thermoreflectance study of the direct energy gap of GaSb", J. Solid state communications, 104 (2): pp. 81-84, 1997. https://doi.org/10.1016/S0038-1098(97)00277-9

[19] T. Moss, "Relations between the refractive index and energy gap of semiconductors", J. physica status solidi, 131 (2): pp. 415-427, 1985. https://doi.org/10.1002/pssb.2221310202

[20] M. Anani, C. Mathieu, S. Lebid, Y. Amar, Z. Chama and H. Abid, "Model for calculating the refractive index of a III-V semiconductor", 41 (4): pp. 570-575, 2008. https://doi.org/10.1016/j.commatsci.2007.05.023 